Preprint (Accepted version) of

Blume, S. O. P., Ben-Mrad, R., \& Sullivan, P. E. (2015). Characterization of coplanar electrode structures for microfluidic-based impedance spectroscopy. Sensors and Actuators B: Chemical. doi:10.1016/j.snb.2015.04.106

\title{
Characterization of coplanar electrode structures for microfluidic-based impedance spectroscopy
}

\author{
Steffen O.P. Blume, Ridha Ben-Mrad, Pierre E. Sullivan \\ Department of Mechanical and Industrial Engineering, University of Toronto, Canada
}

\begin{abstract}
Impedance spectroscopy has the potential for label-free integrated electrochemical detection in microfluidic lab-on-a-chip applications. Its capability to identify and discern between surface and bulk processes in solid-liquid systems finds particular use for the detection of biorecognition events or conductivity measurements. The electrochemical transducer can be in the form of interdigitated electrode structures to increase sensitivity. Experimental work was performed to characterize two different transducer designs. Applications included the monitoring of protein films on contact-less interdigitated electrode structures and conductivity detection of droplets on insulated two-electrode structures. The use of electrode passivation eliminated electrode degradation. Experimental results were compared to theoretical analytical models, and were found to closely correlate with one another. The analytical models were used to design the transducer for optimal conductivity detection. The results inform the current research efforts for the development of in-line impedance spectroscopy in digital microfluidics and confirm the use of simple analytical models for the first-order estimation of the frequency response of interdigitated electrode structures.
\end{abstract}




\section{Introduction}

Impedance spectroscopy (IS) is a non-destructive electroanalytical method, that has been used for corrosion studies, coatings evaluation, batteries, fuel cells, and material characterization $[1,2,3]$. IS can characterize the kinetics of bound and free charges in electrochemical cells, as well as desorption and adsorption activities at surfaces in solid-liquid systems. In recent decades IS has grown as a tool in analytical bioelectrochemistry $[4,5,6,7,8]$.

The application of IS in analytical bioelelectrochemistry is mostly concerned with label-free biorecognition processes at electrode surfaces, such that the impedimetric response of the fluid-electrode system is altered by immobilized macromolecules $[4,6,9,10,11,12]$. Yet other applications also include the detection of changes in the bulk fluid $[6,13]$. Biorecognition processes are usually differentiated into the detection of affinity binding events, the analysis of enzyme-modified electrodes, and cell and microorganism recognition.

The development of lab-on-a-chip devices and the associated need to downscale instrumentation for onchip analyte detection and manipulation has driven innovation of miniaturized transducers. Electrochemical transducers have been considered promising for miniaturization and microsystem-level analysis, due to their compatibility with common microfabrication technologies, without a reduction in sensitivity. This observation stands in contrast to optical methods, that suffer from bulky external instruments [14] and loss of sensitivity with decreasing volumes [15]. In addition, electrochemical transducers can be combined with inexpensive instrumentation, and feature low power consumption, low detection limits, and adjustable selectivity $[16,17,18]$.

A number of research groups have accomplished to combine impedance-based electrochemical detection methods with microfluidic systems [19, 20,21], such as microchannels with integrated gold electrodes for impedance measurements in air, deionized water, and saline solutions [22], the use of two sensing electrodes to determine the impedimetric frequency response of different fluids in a digital microfluidic (DMF) device [19], a microfluidic chip for impedance sensing of cancer cell migration [20], an integrated impedance-based sensor for on-chip monitoring of cell growth in a DMF device [21], or insulated carbon microelectrodes for the monitoring of binding events between BSA and anti-BSA antibodies in microchannels [23].

Interdigitated electrode structures distribute the detection of localized changes to a larger sensing surface $[4,24,25]$. The integration of interdigitated electrode structures into microfluidic systems has seen developments toward impedimetric sensing of protein affinity binding behavior [26], dielectric spectroscopy 
with contact-less microsensors to detect viruses [27] or perform quantitative cell analysis [14], the detection of hormone active chemicals by amperometric monitoring of anodic current and redox cycling between electrode fingers [28], detection of infectious parasites in water and determining their concentration by electrochemical impedance spectroscopy [13], and impedimetric detection of bacterial cells using antimicrobial peptides immobilized onto a microsensor array [12], to list only a few examples. Interdigitated electrode structures are not restricted to the detection of surface effects, but can also be used for bulk conductivity measurements. Theoretical and experimental work on optimizing the structure for conductivity detection has for example been addressed in $[29,30,31,32]$. Applications of conductivity detection included the use of potentiostats and microsensors for $\mathrm{pH}$ and conductivity measurements in a serpentine channel interface [33], and microfluidic devices with dual capacitively coupled contactless conductivity detection by impedance spectroscopy [34].

When immersed in a liquid containing an electroactive target of interest, the metallic electrodes can either be in direct contact with the liquid, or insulated, i.e. contact-less. The use of contact-less electrodes eliminates faradaic processes, which could be desired in the presence of an electrode-coupled redox probe, but can also be unfavorable. Exposed electrodes can suffer from measurement interferences, due to electrode polarization, electrode fouling, and electrolysis [14]. A dielectric insulator can prevent these undesired reactions. On the downside, it has ramifications on the sensor sensitivity. The design of the transducer shifts towards high performing passivation layers that are as thin as possible, have a high electric permittivity, and in the case of affinity binding have the appropriate molecular structure to immobilize biological probes. In this regard, advanced passivation geometries were evaluated by [35], based on three-dimensional numerical simulations to increase sensitivity of interdigitated capacitors and resulted in electric flux density guides and barriers.

While numerical models can provide an accurate representation of the physical, chemical, and electrodynamic characteristics of an electrochemical cell, they impose high computational costs on the design and optimization process. Therefore, inexpensive analytical solutions that can estimate the impedimetric coupling between the electrode fingers, and are subject to simplifying assumptions about the structure of the cell, present as useful surrogates for numerical simulations under certain conditions. Such models have been discussed in $[36,37,38]$.

Presented here are experimental results with simple contact-less interdigitated electrode structures and impedance spectroscopy to study conformation changes in the sensitive region above the electrode fingers due to the presence of a hydrated protein film. The results are compared to values from an analytical 
model for interdigitated electrode structures. This work also presents a two-electrode contact-less structure for conductivity sensing and discusses an approach for the optimization of conductivity cells based on the same analytical model. It was found that the analytical models correlated well with the experimental data and are therefore helpful tools in the first-order estimation of the impedance of interdigitated electrode structures and conductivity cells. 


\section{Test device fabrication}

Test devices were fabricated in the facilities of the University of Toronto Nanofabrication Centre (TNFC). Chromium coated glass substrates were purchased from Deposition Research Lab, Inc. (St. Charles, MO, USA). The substrates were made of $50 \mathrm{~mm} \times 75 \mathrm{~mm} \times 1.1 \mathrm{~mm}$ borosilicate glass slides coated with a $100 \mathrm{~nm}$ chromium layer. An additional $50 \mathrm{~nm}$ gold layer was deposited using a custom TES12D Thermal Evaporator. The gold target material (99.999\% purity gold pellets) was purchased from Kurt J. Lesker Company (Clairton, PA, USA). Typically, chromium adhesion layers are considerably thinner $(\sim 5 \mathrm{~nm})$ than in this case, in which the available chromium coated glass slides were repurposed. The substrates were patterned using standard photolithographic fabrication techniques. Photomasks were made of high resolution films with a minimum feature size of $13 \mu \mathrm{m}$ (Pacific Arts \& Designs, Markham, ON, Canada). Experience with the fabrication of devices had shown that a minimum feature size of $20 \mu \mathrm{m}$ was more feasible.

The substrates were cleaned in an acetone (Fisher Scientific, Pittsburgh, Pennsylvania, USA) bath (5 minutes) and washed in methanol (Fisher Scientific, Pittsburgh, Pennsylvania, USA) (2-3 minutes). Next, the substrates were rinsed in deionized water (DIW), dried with a nitrogen spray gun, and dehydrated on a hot plate for at least 5 minutes at $110^{\circ} \mathrm{C}$. An approximately $12.5 \mathrm{k} \AA$ thin layer of Shipley S1811 photoresist (Marlborough, MA, USA) was spin coated (3000 rpm for 30 seconds), followed by solvent-removal baking for 2 minutes at $110^{\circ} \mathrm{C}$. After exposure in a Suss MicroTec MA6 mask aligner (Garching, Germany) for 7.5 seconds (flood exposure), the substrates were developed in MF-312 (Rohm \& Haas, Dow, Midland, Michigan, USA) for approximately 30 seconds. The substrates were again rinsed in DIW, dried with nitrogen, and post-baked for 1 minute at $115^{\circ} \mathrm{C}$. The substrates were next gold-etched in potassium iodide (Transene Company, Inc., Danvers, Massachusetts, USA) for no longer than 10 seconds to prevent overetching. The etching was stopped by rinsing in DIW and spraying the substrates with nitrogen. The slides were then placed in a chromium etch bath with CR-7S10 etchant (Cyantek Corp., Fremont, California, USA) for approximately 90 seconds. The bath was agitated to obtain a more uniform etch rate across the entire substrate. The slides were again rinsed in DIW and dried with a nitrogen spray. The remaining photoresist was stripped in AZ-300T (Branchburg, New Jersey, USA) while slightly agitating the bath for at least 10 minutes. The slides were DIW-Nitrogen treated and cleaned in acetone and methanol. The substrates were dehydrated on a hot plate at $110^{\circ} \mathrm{C}$ for 5 minutes after being rinsed in DIW and dried with a nitrogen 
spray gun. A $1 \mu \mathrm{m}$ Parylene C layer was vacuum deposited in a Specialty Coating Systems Labcoater (PDS 2010) (Indianapolis, IN, USA). The final step was to coat the substrates with an amorphous fluoropolymer. Teflon AF-1601S (DuPont, Mississauga, ON, Canada) solution was diluted to 1.5 wt\% in Fluorinert FC-40 (Sigma-Aldrich, St. Louis, Missouri, USA). A thin film of approximately $50 \mathrm{~nm}$ was spin coated in a two-step process (Step 1 - Ramp up: 2000 rpm, 20 seconds; Step 2: 2500 rpm, 60 seconds). The substrates were then baked for 20 minutes at $175^{\circ} \mathrm{C}$.

\section{Experimental apparatus}

Images were recorded with an Optronics Macrofire monochrome camera (Muskogee, Oklahoma, USA) installed on a Zeiss SteREO Discovery.V12 microscope (Jena, Germany). Impedance measurements were acquired with an Agilent Technologies E5061B ENA Series Network Analyzer (Santa Clara, California, USA) with an impedance analysis option. The network analyzer was configured in the Port 1-2 Series mode, configuring the two S-parameter terminals (port 1 and 2) of the analyzer to be used for impedance measurements with a frequency sweep from $5 \mathrm{~Hz}$ to $300 \mathrm{MHz}$. Before each measurement series the analyzer was calibrated using a non-inductive high precision through-hole $50 \Omega$ resistor, and was connected to the test devices via BNC cables and alligator connectors.

The electrochemical cell can be considered as a system that takes an input signal and transfers it into an output signal [39]. Most if not all systems are nonlinear. It is however possible to use a linear approximation for most systems, especially if the input signal amplitude is small. The input to output dependence of a nonlinear, time invariant system around some operation point $x_{0}$ can be expressed in terms of a Taylor series expansion $[40]$

$$
y(x)=y\left(x_{0}\right)+\left.\frac{\partial y(x)}{\partial x}\right|_{x=x_{0}}\left(x-x_{0}\right)+\left.\sum_{k=2}^{\infty} \frac{1}{k !} \frac{\partial^{k} y(x)}{\partial x^{k}}\right|_{x=x_{0}}\left(x-x_{0}\right)^{k}
$$

Considering an input signal of the form $x(t)=x_{0}+\Delta x$, where the variation amplitude of the signal $\Delta x$ is small, it is acceptable to ignore any higher order terms (quadratic and higher) and assume that the system therefore is quasi-linear.

Hence, for small signal amplitudes on the order of $10 \mathrm{mV}$, an electrochemical cell can be assumed to behave linearly. The advantage of such a linear system is that process characteristics such as diffusion rates can be more accurately determined. The problem with low amplitude signals is that background noise and parasitic contributions outside of the electrochemical cell can detrimentally interfere with the 
signal. Since the network analyzer is somewhat constrained in its function as an impedance analyzer, the measurement signal was adjusted to $707 \mathrm{mV}$. It should however be noted that this can affect linearity and results need to be more cautiously analyzed. Since the measurements were gathered solely on the grounds of detecting changes in the impedance spectra and were not further processed to determine specific reaction characteristics, error estimates due to potential non-linearities are not included here.

Electrolyte solutions were prepared by dissolving potassium chloride $(\mathrm{KCl})$ powder $\left(\right.$ BioShop $^{R}$ Canada Inc, Burlington, ON, Canada) in distilled deionized water (Mediatech, Inc. Manassas, VA, USA) with five different concentrations: $0.0001 \mathrm{M}, 0.001 \mathrm{M}, 0.01 \mathrm{M}, 0.1 \mathrm{M}$, and $1 \mathrm{M} \mathrm{KCl}$. Bovine serum albumin (BSA) solutions were prepared by diluting BSA at $0.01,0.05,0.1,0.5$, and $1 \%(\mathrm{w} / \mathrm{v})$ in distilled deionized water.

\section{Design of interdigitated electrode structures}

For the materials and fabrication constraints used, it was shown in previous work [38] that an interdigitated electrode structure with 24 fingers is expected to perform best with regard to sensitive detection of surface effects. An example of a 24-electrode interdigitated electrode structure is sketched in Figure 1(a). The total array size was limited to approximately $1 \times 1 \mathrm{~mm}^{2}$. The electrode finger width $\mathrm{w}$, and inter-electrode gap size $g$ were roughly $20 \mu \mathrm{m}$. The comb-like electrodes were each connected to one of the two terminals of the network analyzer via bond pads, creating alternating signals between the interdigitated fingers.

Figure 1 illustrates a fabricated interdigitated electrode test structure. Droplets of approximately 5 $\mu \mathrm{l}$ volume were manually dispensed on the sensor electrodes. Before a new droplet could be dispensed, the droplets were removed with a syringe. Because of the hydrophobic coating on the transducer and the capillary pull force of the syringe tip, the fluid could be removed without wiping the sensor surface, avoiding any damage to the transducers.

\section{Results and discussion}

The performance of different transducer designs for the detection of surface effects and bulk properties was evaluated two ways:

1. Studying the impedance change due to a hydrated protein film evaporated onto a 24 -finger interdigitated electrode structure;

2. Analyzing the frequency response for various bulk conductivities of water-based solutions on a twoelectrode structure 


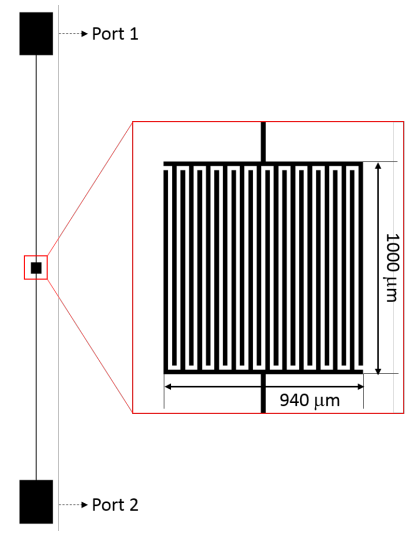

(a) Sketch of a test device showing the bond pads and interdigitated electrode fingers $(N=24)$.

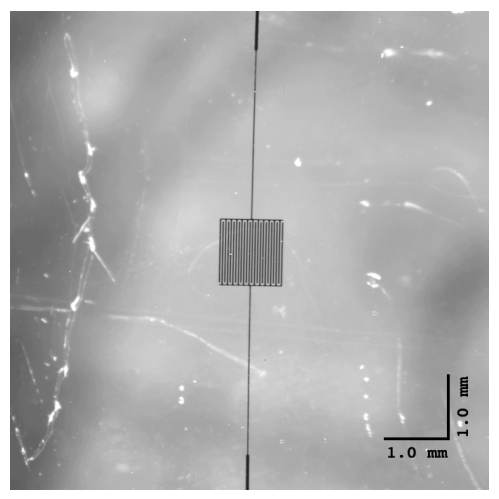

(b) 24-electrode test device

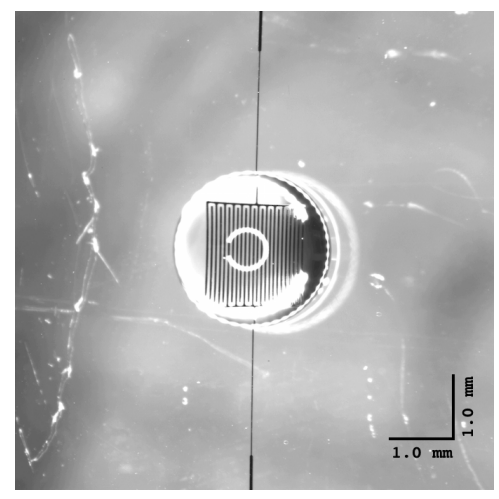

(c) Droplet wetting the surface of a $24-$ electrode test device

Figure 1 Test devices. The electrodes are patterned on a borosilicate glass substrate. Parylene C and Teflon AF1601 provide electric insulation and a hydrophobic surface. Droplets were manually dispensed for sensor characterization. The visible scratches in (b) and (c) are on the backside of the transparent glass substrates and occurred during handling.

In both cases the experimental results were compared to analytical model predictions with the material properties listed in Table 1.

\section{Hydrated protein film detection}

An important feature of interdigitated electrode structures is the sensitive detection of processes occurring at the transducer-analyte interface, such as adsorption of biological probes on the transducer surface. Ultimately, this translates into changes in the physico-chemical states and conformation of the electrochemical cell.

To test the frequency response changes of a 24 -finger interdigitated electrode structure as a result of the growth of protein films on the transducer surface, the impedance was initially measured in an open environment exposed to air. This reference measurement served for comparison in the proceeding protein deposition test, in which a protein film was created by droplet drying. A $5 \mu$ l droplet of the BSA stock solution $(1 \%(\mathrm{w} / \mathrm{v}))$ was dispensed on the transducer surface. While the water content of the droplet was evaporating, the impedance was measured approximately every two minutes. The last measurement data was recorded after a stable residual film of hydrated BSA remained. The goal was to observe a change in the impedance spectrum between the first and last measurement, namely with and without the BSA film, 
Table 1 Material properties assumed to model the frequency response of a multi-layer interdigitated electrode structure.

\begin{tabular}{|c|c|c|c|c|}
\hline Material & Thickness & $\begin{array}{l}\text { Relative } \\
\text { permittivity, } \varepsilon_{r}\end{array}$ & $\begin{array}{l}\text { Conductivity, } \\
\sigma\left(\mathrm{S} \cdot \mathrm{m}^{-1}\right)\end{array}$ & References \\
\hline \multicolumn{5}{|c|}{ Transducer substrate and passivation } \\
\hline Borosilicate glass & $t \rightarrow \infty$ & 4.1 & - & {$[41]$} \\
\hline Parylene $\mathrm{C}^{*}$ & $t=1 \mu \mathrm{m}$ & 3.05 & $1.0 \times 10^{-8}$ & {$[42]$} \\
\hline Teflon ${ }^{\circledR}$ AF1601** & $t=50 \mathrm{~nm}$ & 1.93 & - & {$[43]$} \\
\hline \multicolumn{5}{|l|}{ Protein film test } \\
\hline Bovine serum albumin & variable & $\varepsilon_{r}=f\left(c_{\mathrm{H}_{2} \mathrm{O}}\right)$ & $\sigma=f\left(c_{\mathrm{H}_{2} \mathrm{O}}\right)$ & Table 2 \\
\hline Air & $t \rightarrow \infty$ & 1.0 & - & - \\
\hline \multicolumn{5}{|l|}{ Conductivity test } \\
\hline Electric double layer & $t=f\left(c_{K C l}\right)$ & 80.1 & - & Equation (2) \\
\hline Electrolyte solution & $t \rightarrow \infty$ & 80.1 & $\sigma=f\left(c_{K C l}\right)$ & Table 3 \\
\hline \multicolumn{5}{|c|}{$\begin{array}{l}\text { * The surface resistivity of Parylene } \mathrm{C} \text { is } 1.0 \times 10^{14} \Omega[42] \text {. Given that the thickness of the insulator film is } 1 \mu \mathrm{m} \text {, } \\
\text { the equivalent conductivity becomes } \sigma=1.0 \times 10^{-8} \mathrm{~S} \cdot \mathrm{m}^{-1} \text {. }\end{array}$} \\
\hline
\end{tabular}

mimicking the growth and adsorption of biological targets on the transducer surface.

Figure 2 is the resulting frequency response in terms of the amplitude of the impedance. As the droplet gradually evaporates, the impedance spectrum first drops and after 32 minutes recedes upward toward the curve corresponding to the open measurement, as indicated by the dashed arrow in Figure 2(a). The fact that the curve jumps back toward the open measurement indicates that the water content and droplet size reduced past a critical level to have significant impact on the impedance after 32 minutes. Instead, the conformation and hydration level of the deposited protein film become more dominant. The curves in Figure 2(b) show a small difference of two orders of magnitude between the reference measurement and the final protein film measurement. The measured frequency response was compared to the analytical model in [38]. The transducer structure consists of stratified layers as shown in Figure 3. On top of the insulated interdigitated electrode structure exists a thin homogeneous layer with a uniform thickness of hydrated BSA. The relative permittivity and conductivity of the BSA film both depend on the level of hydration. In addition, the relative permittivity is also a function of the applied frequency, yet becomes roughly constant for frequencies higher than $1 \mathrm{kHz}$ [45]. Table 2 compiles the relative permittivity and conductivity of hydrated BSA at different hydration levels. The exact hydration level of the BSA film was 


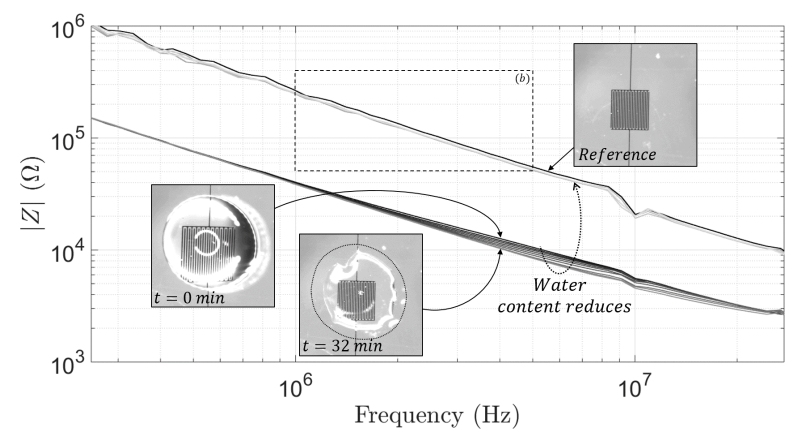

(a)

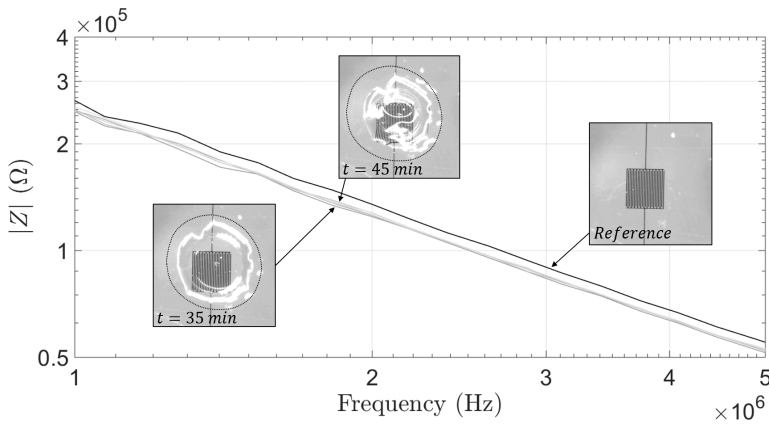

(b)

Figure 2 Bode magnitude plot of impedance measurements performed to determine changes of the layer conformation above a multi-finger electrode array $(w=g=20 \mu \mathrm{m}, L=1 \mathrm{~mm}, N=24)$. The box frame in (a) indicates the data range shown in (b). The inserted images show sequences of a droplet (1\% $(w / v)$ BSA in deionized water) gradually evaporating and leaving behind a thin protein film. The dashed lines in the insets indicate the approximate original droplet size.

unknown, but was assumed to be below $20 \%$. Since the goal was to obtain a first-order estimate of the expected impedance, two scenarios are considered, each with its own simplifying assumptions about the hydration level and thickness of the BSA film:

1. The film thickness is fixed at $1 \mu \mathrm{m}$ and the impedance is determined for the hydration levels and corresponding electrical properties given in Table 2;

2. The second case assumes $10 \%$ hydration and five different film thicknesses $(10 \mathrm{~nm}, 100 \mathrm{~nm}, 1 \mu \mathrm{m}, 10$ $\mu \mathrm{m}$, and $100 \mu \mathrm{m})$.

The resulting modeled impedance spectra from $1 \mathrm{MHz}$ to $5 \mathrm{MHz}$ are shown in Figures 4(a) and 4(b) for case one and two, respectively. The frequency range is the same as for the measured impedance spectrum shown in Figure 2(b). Figure 4 demonstrates that the impedance is expected to be more sensitive to the height of the BSA film than the hydration level. When comparing Figure 4 and Figure 2(b), it can be noted that the analytical model accurately predicts the open reference measurement. In addition, the impedance of the final protein film measurement was approximately two orders of magnitude smaller than the reference measurement. The model confirms this observation and helps to estimate the thickness and hydration level of the actual BSA film. Based on Figure 4(a), the magnitude of the impedance is relatively invariable to changes in the hydration of the BSA film. Assuming a hydration level between $10 \%$ and $20 \%$ is therefore reasonable. Hence, based on the results shown in Figure 4(b), the film thickness can be 


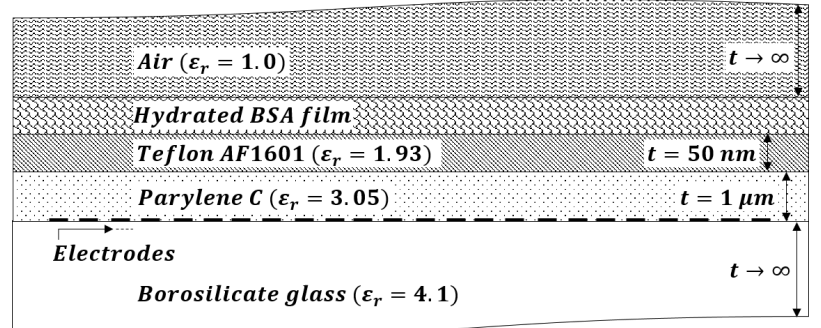

Figure 3 Assumed cross section of the interdigitated electrode structure with a BSA film coated across the transducer surface. The electrode fingers $(N=24)$ are patterned on a borosilicate glass substrate. Parylene $C$ and Teflon AF1601 provide electric insulation and a hydrophobic surface between the BSA film and the electrode fingers. The assumed thickness and material properties of each layer is indicated. Material properties of the BSA film are listed in Table 2. The electrode finger width and inter-electrode gap size are $20 \mu \mathrm{m}$.

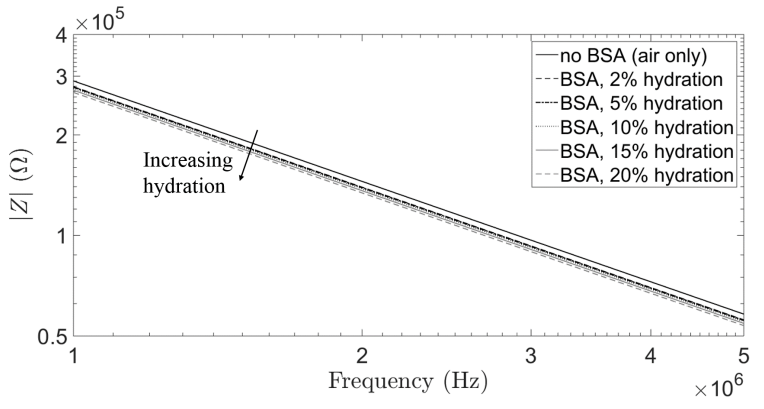

(a) Case 1

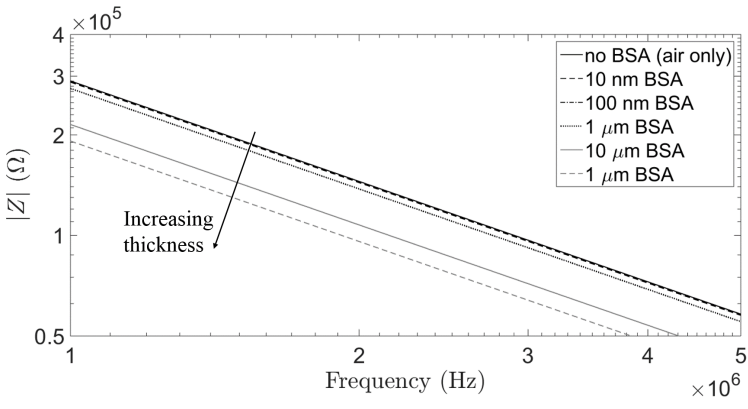

(b) Case 2

Figure 4 Bode magnitude plot of the modeled frequency response of a multi-finger electrode array coated with a thin film of BSA based on the analytical model discussed in [38]. The model assumes the simplified structure shown in Figure 3 to derive the impedance of the electrochemical cell. In (a) the BSA film thickness is assumed to be $1 \mu \mathrm{m}$. In (b) the hydration level is fixed at $10 \%$.

estimated to be roughly $1 \mu \mathrm{m}$.

\section{Conductivity measurements}

A top view of the test device is shown in Figure 5. Water-based droplets with different concentrations of $\mathrm{KCl}$, and volumes of approximately $5 \mu \mathrm{l}$ were manually dispensed on the transducer surface. The graphs in Figure 6 present the frequency response for various electrolyte concentrations averaged over four measurements for each concentration. The solid black lines show the average value of the four measurements. The grey shaded areas indicate the spread between the minimum and maximum values measured. The impedance spectrum is distorted at both the low-frequency (high impedance) and high-frequency (low impedance) bands. This was 
Table 2 Conductivity and relative permittivity of hydrated BSA [45].

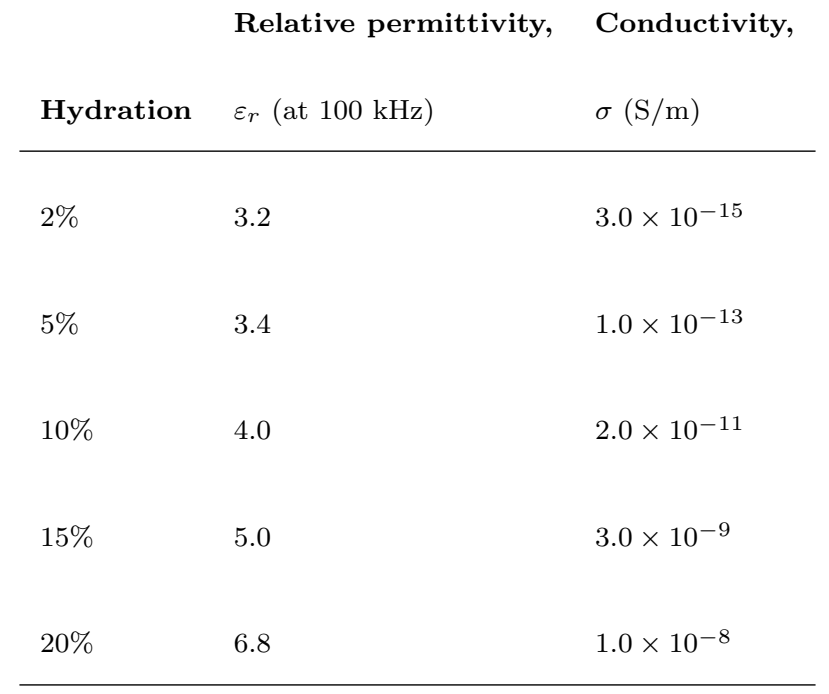

because the network analyzer was unable to precisely resolve high and low impedance values. Nonetheless, the mid-to-high frequency values reveal clear distinctions between the different electrolyte solutions. Figure 7 shows the geometry of the conductivity cell, consisting of an insulating Parylene C layer, a hydrophobic Teflon ${ }^{\circledR}$ fluoropolymer layer, the electric double layer, and the bulk electrolyte solution. The thickness of the electric double layer $x_{d l}$ depends on the radius of hydrated ions and the applied potential. It can be approximated by [46]

$$
x_{d l}=1.5 \kappa^{-1}
$$

where $\kappa^{-1}$ is the Debye length (see Appendix A), which depends on the concentration of the electrolyte. For non-conducting surfaces without redox reactions, charge transfer between the electrodes and the fluid is not present. Therefore, the solid-liquid interface is only characterized by the charge distribution within the electric double layer. It is modeled as a purely dielectric layer with a permittivity equal to that of the bulk electrolyte solution.

The conductive bulk solution can be described by the complex, frequency-dependent, relative permittivity,

$$
\varepsilon_{r}^{*}=\varepsilon_{r}-j \frac{\sigma}{\varepsilon_{0} \omega}
$$

where $\varepsilon_{r}$ and $\sigma$ are the relative permittivity and conductivity of the material, respectively. When using the analytical model [38], the relative permittivity is replaced by the complex relative permittivity, and the 


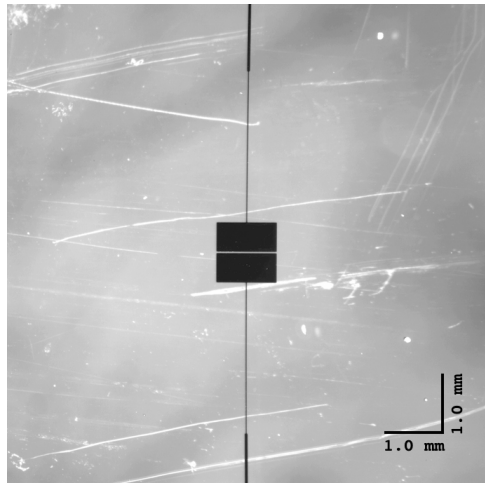

(a) Two-electrode test device

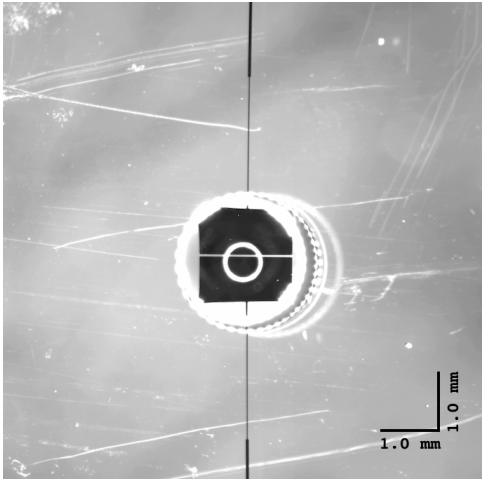

(b) Droplet wetting the surface of the test device

Figure 5 Top-down view of a test device consisting of two electrode fingers, with a spacing of $20 \mu m$. The finger width and length is $490 \mu \mathrm{m}$ and $1 \mathrm{~mm}$, respectively. The electrodes are patterned on a borosilicate glass substrate. Parylene $C$ and Teflon AF1601 provide electric insulation and a hydrophobic surface. The visible scratches are on the backside of the transparent glass substrates and occurred during handling.

assumed boundary conditions specified at the layer interfaces need to comply with both the permittivity and conductivity gradients.

Figure 8 shows the Bode diagram based on the analytical model. The model parameters for the glass substrate, insulator, and fluoropolymer layer are given in Table 1. The solution conductivities of different $\mathrm{KCl}$ concentrations are compiled in Table 3 in Appendix B. The cell is connected in series with a resistor and inductor, accounting for the resistance $(\approx 800 \Omega)$ and inductance $\left(\approx 3.0 \times 10^{-8} \mathrm{H}\right)$ of the lead wires.

The modeled impedance spectrum shows a similar behavior as the measured impedance. Constant impedance plateaus and phase angle crests occur where conductive control becomes dominant over the otherwise dielectric behavior. The low-frequency plateau is associated with the sheet resistance of the insulator, while the mid-to-high frequency plateaus are associated with the bulk resistance of the different electrolyte solutions. At high frequencies, the resistance of the lead wires prevails. The ideal conductivity of pure water is $5.5 \times 10^{-6} \mathrm{~S} \cdot \mathrm{m}^{-1}$. The model used a conductivity of $4.0 \times 10^{-4} \mathrm{~S} \cdot \mathrm{m}^{-1}$ to better match the measured response. A possible reason for the increased conductivity of water could be the contamination of reagent tubes used to store the liquid.

The modeled amplitude is slightly offset from the measured amplitude, which could be due to threedimensional fringing field effects on the ends of the electrodes, which are not captured by the model. 


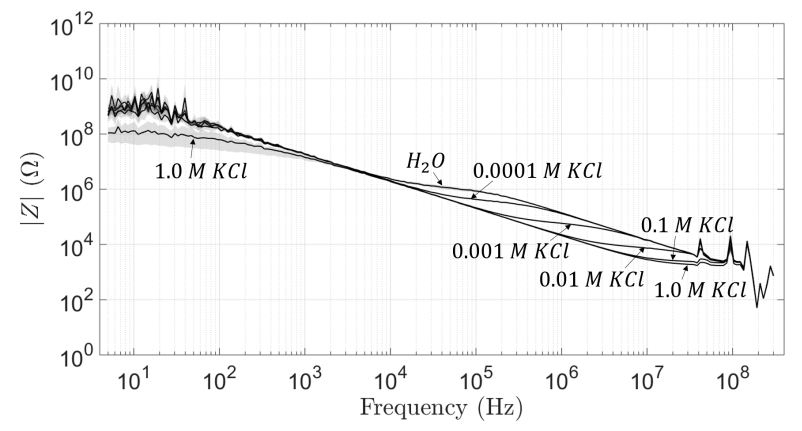

(a) Modulus

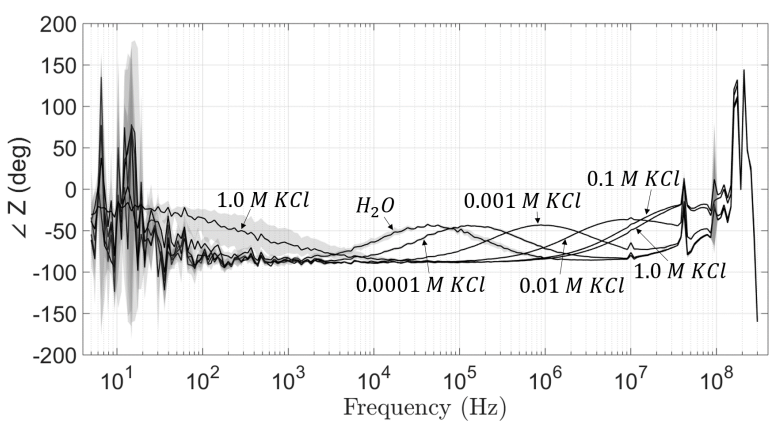

(b) Phase

Figure 6 Bode plot of frequency response of a two-electrode conductivity sensor.

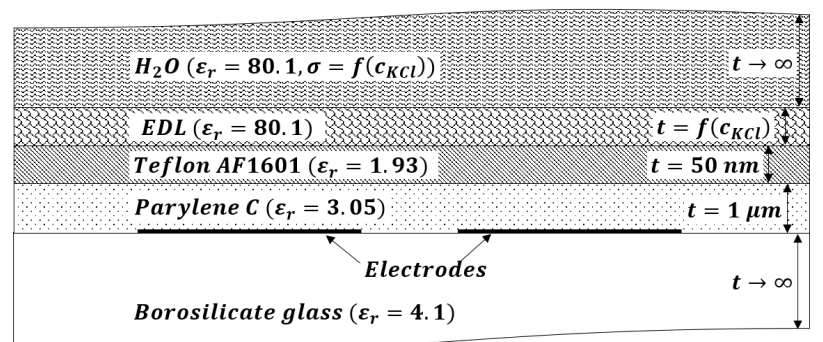

Figure 7 Assumed cross section of conductivity cell with insulated electrodes. The electrodes are patterned on a borosilicate glass substrate. Parylene $C$ and Teflon AF1601 provide electric insulation and a hydrophobic surface between the electrolyte solution and the electrodes. The assumed thickness and material properties of each layer are indicated. The thickness of the electric double layer is a function of the electrolyte concentration.

Typically these three-dimensional effects are negligible for very long electrode fingers, whereas here the electrode width and length is approximately of the same order of magnitude.

A noticeable feature in the measured frequency response is the particularly pronounced low-frequency plateau for a $\mathrm{KCl}$ concentration of $1.0 \mathrm{M}$. The concentration of ions at the electrolyte-surface interface is larger than in the bulk solution. The ensuing concentration gradient can cause diffusion of ions overshadowing the voltage-driven ionic current. With lumped-parameter modeling, these low-frequency diffusion effects are typically modeled by the Warburg impedance [2]. The observation that this behavior is distinctly prevalent for $1.0 \mathrm{M} \mathrm{KCl}$ electrolyte solutions, yields that high electrolyte concentrations presumably cause stronger ion concentration gradients at the liquid solid interface, measurable as more pronounced Warburg impedance effects and stronger diffusion control in the low-frequency regions, which cannot be captured by the analytical model. 


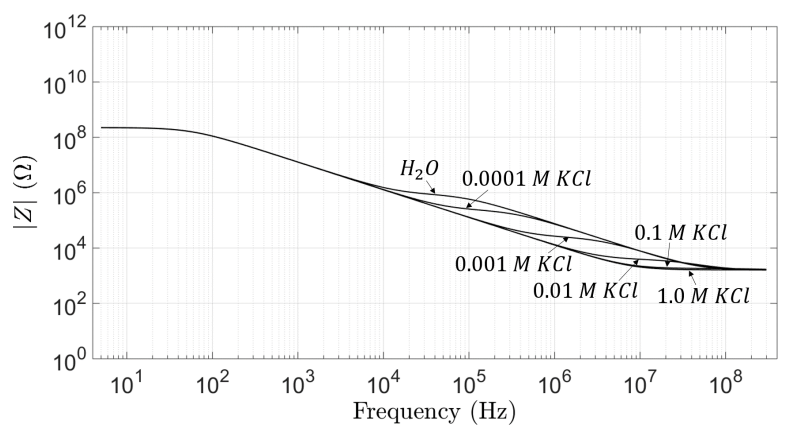

(a) Modulus

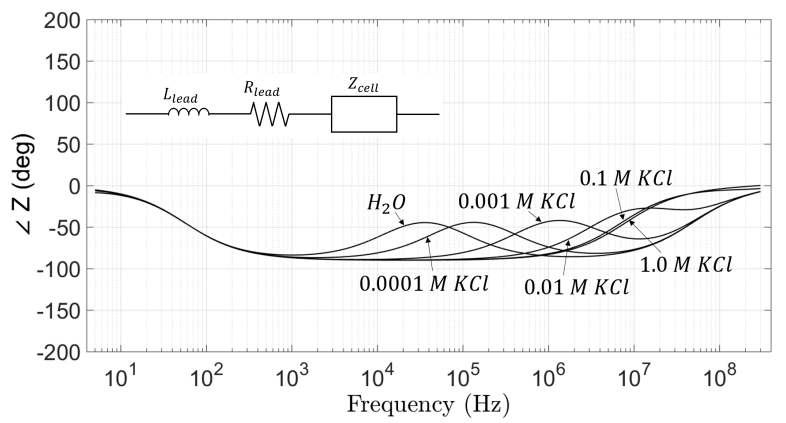

(b) Phase

Figure 8 Modeled frequency response for a two-electrode conductivity sensor based on the analytical model discussed in [38]. The model assumes the simplified structure shown in Figure 7 to derive the impedance of the electrochemical cell $Z_{\text {cell }}$. The inset in (b) illustrates the lumped circuit elements used to represent additional resistive and inductive contributions particularly dominant in the high-frequency domain.

\section{Detecting bulk protein concentrations}

The conductivity of water changes as a function of the concentration of BSA [47]. Water-based droplets $(\approx 5 \mu \mathrm{l})$ with different concentrations of BSA were dispensed on the transducer surface. Before every new sample the transducer surface was washed with a deionized distilled water solution containing $0.05 \%$ Tween-20. The tested transducer was similar to the one illustrated in Figure 5, composed of two electrodes each with a width of $490 \mu \mathrm{m}$ and separated by a gap of $20 \mu \mathrm{m}$. The length of the electrodes was $1 \mathrm{~mm}$.

Figure 9 shows the Bode impedance plot for deionized distilled water solutions with different concentrations of BSA. Since BSA affects the conductivity of the bulk solution, the location of the constant impedance plateau changes depending on the protein concentration. The graphs for concentrations higher than $0.05 \%$ $(\mathrm{w} / \mathrm{v})$ are easily distinguishable. The plateaus move to lower impedance values for increasing protein 


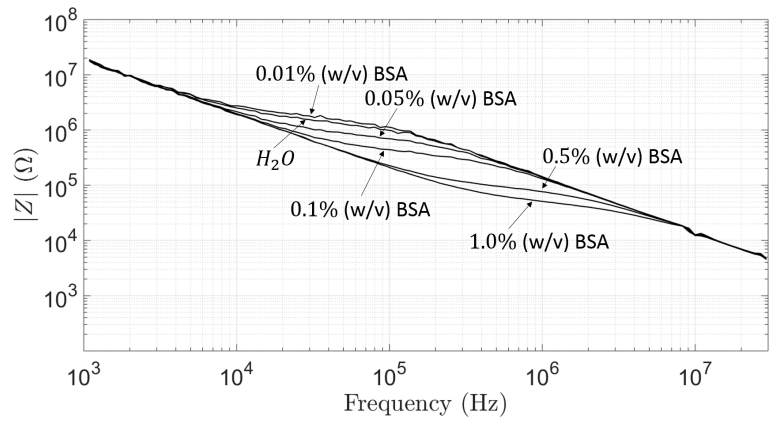

(a) Modulus

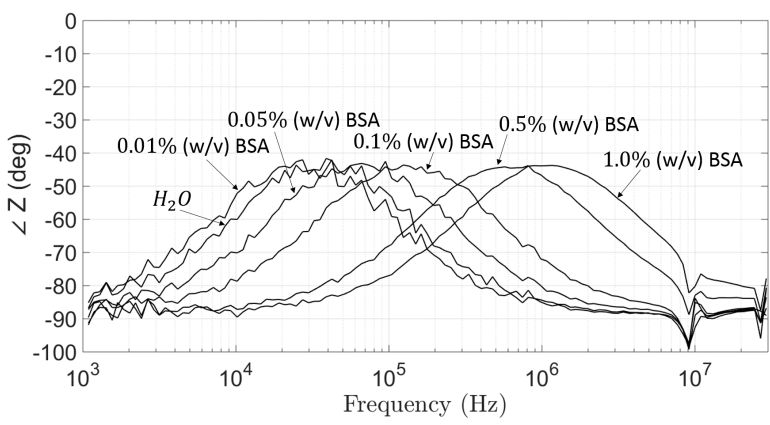

(b) Phase

Figure 9 Bode plot of frequency response for deionized water with different concentrations of BSA.

concentrations, i.e. the crest in the Bode phase plot moves to the right, indicating that the conductivity increases with increasing concentration. Also, the sensitivity to changes in the protein concentration is a relative function of the protein concentration itself. Unless a more rigorous analysis with larger as well as denser sampling ranges is performed, quantifiable results about the sensitivity as well as other performance characteristics such as detection limits are not available.

\section{Optimizing electrode geometry for conductivity detection}

Optimization of of conductivity cells for sensitive detection of the solution resistance has been discussed by $[30,31,29,32]$, using conformal mappings to determine the cell constant of coplanar electrode structures, yet with less attention to multiple layers and different material properties.

For an ideal conductivity cell the width of the constant-impedance plateau is maximized. The analytical model discussed in [38] can predict the behavior of a conductivity cell and can be used to optimize for a maximum plateau width. The structure of the cell is assumed as shown in Figure 7. The material properties are given in Table 1. The goal is to explore the possible combinations of electrode width w, gap distance $g$, and number of electrode fingers $N$, as well as determine the frequency response for each combination. To reduce the optimization parameters, the metalization ratio is introduced, given by $\eta=\mathrm{w} /(\mathrm{w}+g)$. The number of electrode fingers is restricted to only even numbers. For each combination of $\eta$ and $N$, the frequency response can be determined along with the upper and lower bounds of the constant-impedance plateau, denoted as $f_{l o}$ and $f_{h i}$ respectively. Due to the logarithmic scale of the frequency response, the optimization aims to maximize $\Delta f=\log _{10}\left(f_{h i}\right)-\log _{10}\left(f_{l o}\right)$.

Figure 10 shows the result for $\Delta f$ with respect to $\eta$ and $N$. The greyed parts are regions for which the 
minimum feature size constraints $(20 \mu \mathrm{m})$ are not met or the total width of the electrode array exceeds 1 $\mathrm{mm}$. The maximum is achieved for $\eta=0.55$ and $N=2$, resulting in a two-electrode design with $\mathrm{w}=355$ $\mu \mathrm{m}$ and $g=290 \mu \mathrm{m}$.

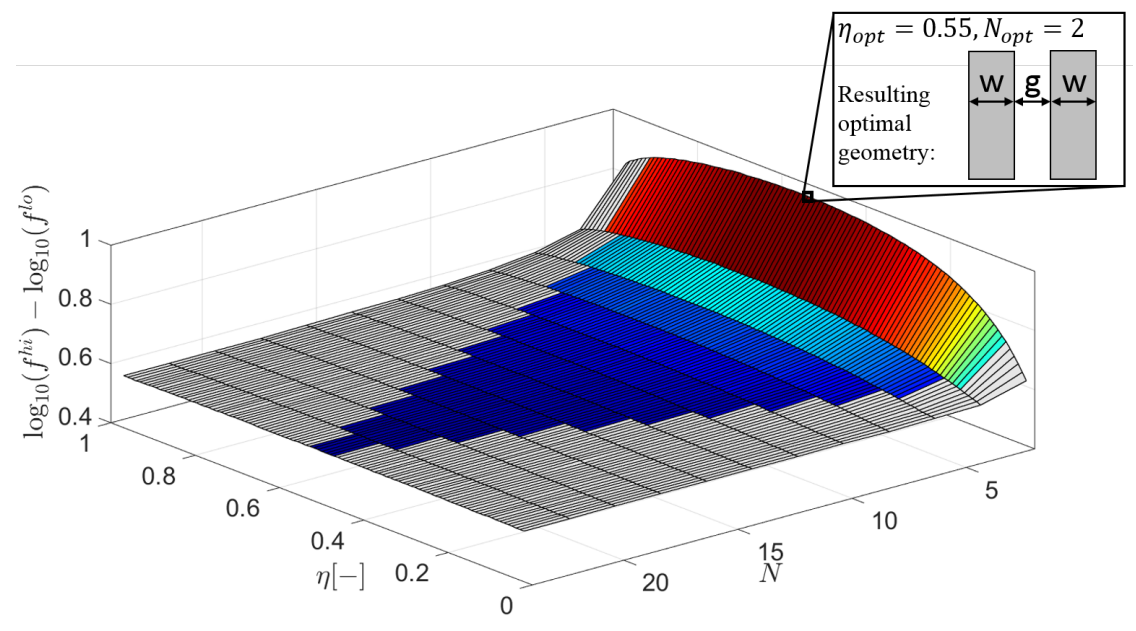

Figure 10 Difference between low and high frequency bound of constant-impedance plateau as a function of the metalization ratio and the number of electrode fingers.

The sensitivity of interdigitated electrode structures to bulk effects, such as the solution conductivity, reduces with increased electrode fingers. Additionally, the allowable transducer footprint limits the possible design variations of electrode finger width and inter-electrode gap.

It should be noted that because the electric double layer is very thin and has a high permittivity compared to the remaining layers, it could have been ignored here, without affecting the results. However, for thin film passivation materials with higher permittivities and possible thinner dimensions, such as silicon dioxide $\left(\mathrm{SiO}_{2}, \varepsilon_{r} \approx 3.7\right)$ or tantalum pentoxide $\left(\mathrm{Ta}_{2} \mathrm{O}_{5}, \varepsilon_{r} \approx 20-25\right)$, the effect of the electric double layer can be important. 


\section{Conclusion}

This work presented the characterization of two- and multi-electrode transducers for possible applications of impedance spectroscopy in microfluidic systems. Depending on the application, the design of the transducer can be augmented for bulk or surface detection in liquid-solid systems. Presented applications included the monitoring of hydrated protein films on insulated interdigitated electrode structures, and conductivity detection of electrolyte and protein solutions on two-electrode structures. The use of passivation layers prevented electrode degradation and thus promotes repeatable measurement conditions. Due to simple fabrication techniques and the use of materials that can typically be found in DMF, the transducers are expected to be particular compatible with DMF lab-on-a-chip devices and hence suitable for in-line contactless bioelectrochemical detection. Results from simple analytical models correlated well with the measured impedimetric frequency response of the test devices. The theoretical results were used in predicting and investigating the measured response, and optimizing the transducer design for more sensitive conductivity detection. The analytical models can be used to inform the design of interdigitated electrode structures for specific applications in microfluidic research. It was shown that for bulk conductivity detection, the sensitivity is highest for two-electrode configurations with a metalization ratio of 0.55 , and that increasing the number of electrode fingers can significantly reduce sensitivity. For surface detection it remains that multi-electrode structures are superior over two-electrode structures.

\section{Acknowledgments}

The authors acknowledge CMC Microsystems for the provision of products and services that facilitated this research, including the Agilent Technologies E5061B ENA Series Network Analyzer, the Natural Sciences and Engineering Research Council of Canada (NSERC) and the Ontario Centres of Excellence (OCE) for support through their granting programs. 


\section{References}

[1] J. Ross Macdonald. Impedance Spectroscopy. Annals of Biomedical Engineering, 20:289-305, 1992.

[2] Evgenij Barsoukov and J. Ross Macdonald. Impedance Spectroscopy: Theory, Experiment, and Applications. John Wiley \& Sons, Inc., Hoboken, New Jersey, 2 edition, 2005.

[3] Utz Retter and Heinz Lohse. Electrochemical Impedance Spectroscopy. In Fritz Scholz, editor, Electroanalytical Methods: Guide to Experiments and Applications, chapter II.5, pages 159-176. Springer-Verlag, Berlin, Heidelberg, Germany, 2 edition, 2010 .

[4] Eugenii Katz and Itamar Willner. Probing Biomolecular Interactions at Conductive and Semiconductive Surfaces by Impedance Spectroscopy: Routes to Impedimetric Immunosensors, DNA-Sensors, and Enzyme Biosensors. Electroanalysis, 15(11):913-947, 2003.

[5] Jian-Guo Guan, Yu-Qing Miao, and Qing-Jie Zhang. Impedimetric biosensors. Journal of bioscience and bioengineering, 97(4):219-26, January 2004. ISSN 1389-1723. doi: 10.1016/S1389-1723(04)70195-4. URL http://www.ncbi.nlm.nih.gov/ pubmed/16233619.

[6] Isaac O. K'Owino and Omowunmi A. Sadik. Impedance Spectroscopy: A Powerful Tool for Rapid Biomolecular Screening and Cell Culture Monitoring. Electroanalysis, 17(23):2101-2113, December 2005. ISSN 1040-0397. doi: 10.1002/elan.200503371. URL http://doi.wiley.com/10.1002/elan.200503371.

[7] Jonathan S. Daniels and Nader Pourmand. Label-Free Impedance Biosensors: Opportunities and Challenges. Electroanalysis, 19(12):1239-1257, May 2007. ISSN 1040-0397. doi: 10.1002/elan.200603855. URL http://www.pubmedcentral.nih. gov/articlerender.fcgi? artid $=2174792 \&$ tool $=$ pmcentrez\&rendertype=abstract .

[8] Byoung-Yong Chang and Su-Moon Park. Electrochemical impedance spectroscopy. Annual review of analytical chemistry (Palo Alto, Calif.), 3:207-229, 2010. ISSN 1936-1335. doi: 10.1146/annurev.anchem.012809.102211.

[9] F. Lisdat and D. Schäfer. The use of electrochemical impedance spectroscopy for biosensing. Analytical and Bioanalytical Chemistry, 391(5):1555-1567, 2008. ISSN 16182642. doi: 10.1007/s00216-008-1970-7.

[10] M. Barreiros dos Santos, J.P. Agusil, B. Prieto-Simón, C. Sporer, V. Teixeira, and J. Samitier. Highly sensitive detection of pathogen Escherichia coli O157:H7 by electrochemical impedance spectroscopy. Biosensors 6 bioelectronics, 45:174-80, 2013. ISSN 1873-4235. doi: 10.1016/j.bios.2013.01.009. URL http://www.ncbi.nlm.nih.gov/pubmed/23500360.

[11] Luca Ianeselli, Gianluca Grenci, Carlo Callegari, Massimo Tormen, and Loredana Casalis. Development of stable and reproducible biosensors based on electrochemical impedance spectroscopy: Three-electrode versus two-electrode setup. Biosensors and Bioelectronics, 55:1-6, 2014. ISSN 09565663. doi: 10.1016/j.bios.2013.11.067. URL http: //dx.doi.org/10.1016/j.bios.2013.11.067. 
[12] P. B. Lillehoj, C. W. Kaplan, J. He, W. Shi, and C.-M. Ho. Rapid, Electrical Impedance Detection of Bacterial Pathogens Using Immobilized Antimicrobial Peptides. Journal of Laboratory Automation, 19(1):42-49, 2013. ISSN 2211-0682. doi: 10.1177/2211068213495207. URL http://jla.sagepub.com/lookup/doi/10.1177/2211068213495207.

[13] T. Houssin, J. Follet, A. Follet, E. Dei-Cas, and V. Senez. Label-free analysis of water-polluting parasite by electrochemical impedance spectroscopy. Biosensors and Bioelectronics, 25(5):1122-1129, 2010. ISSN 09565663. doi: 10.1016/j.bios.2009. 09.039 .

[14] P. Ertl and R. Heer. Interdigitated impedance sensors for analysis of biological cells in microfluidic biochips. Elektrotechnik und Informationstechnik, 126(1-2):47-50, February 2009. ISSN 0932-383X. doi: 10.1007/s00502-009-0607-7. URL http://link.springer.com/10.1007/s00502-009-0607-7.

[15] Francesco Ricci, Gianluca Adornetto, and Giuseppe Palleschi. A review of experimental aspects of electrochemical immunosensors. Electrochimica Acta, 84:74-83, December 2012. ISSN 00134686. doi: 10.1016/j.electacta.2012.06.033. URL http://linkinghub.elsevier.com/retrieve/pii/S0013468612009735.

[16] A. Warsinke, A. Benkert, and F.W. Scheller. Electrochemical immunoassays. Fresenius' journal of analytical chemistry, 366(6-7):622-34, 2000. ISSN 0937-0633. URL http://www.ncbi.nlm.nih.gov/pubmed/11225774.

[17] Julia Yakovleva and Jenny Emneus. Electrochemical Immunoassays. In Philip Bartlett, editor, Bioelectrochemistry: Fundamentals, Experimental Techniques and Applications, chapter 11, pages 377-410. John Wiley \& Sons, Inc., 2008. ISBN 9780470843642.

[18] Mamas I. Prodromidis. Impedimetric immunosensors - A review. Electrochimica Acta, 55(14):4227-4233, May 2010. ISSN 00134686. doi: 10.1016/j.electacta.2009.01.081. URL http://linkinghub.elsevier.com/retrieve/pii/S0013468609002138.

[19] Thomas Lederer, Stefan Clara, Bernhard Jakoby, and Wolfgang Hilber. Integration of impedance spectroscopy sensors in a digital microfluidic platform. Microsystem Technologies, 18(7-8):1163-1180, March 2012. ISSN 0946-7076. doi: 10.1007/s00542-012-1464-6. URL http://link.springer.com/10.1007/s00542-012-1464-6.

[20] Tien Anh Nguyen, Tsung-I Yin, Diego Reyes, and Gerald A. Urban. Microfluidic chip with integrated electrical cell-impedance sensing for monitoring single cancer cell migration in three-dimensional matrixes. Analytical chemistry, 85(22):11068-76, November 2013. doi: 10.1021/ac402761s. URL http://pubs.acs.org/doi/abs/10.1021/ac402761s.

[21] Steve C.C. Shih, Irena Barbulovic-Nad, Xuning Yang, Ryan Fobel, and Aaron R. Wheeler. Digital microfluidics with impedance sensing for integrated cell culture and analysis. Biosensors \& Bioelectronics, 42:314-20, April 2013. ISSN 1873-4235. doi: 10.1016/j.bios.2012.10.035. URL http://www.ncbi.nlm.nih.gov/pubmed/23208104.

[22] H. Edward Ayliffe, A. Bruno Frazier, and R.D. Rabbitt. Microchannels with Integrated Metal Electrodes. IEEE Journal of Microelectromechanical Systems, 8(1):50-57, March 1999.

[23] Mohammed Kechadi, Bruno Sotta, Lila Chaal, Bernard Tribollet, and Jean Gamby. A real time affinity biosensor on an insulated polymer using electric impedance spectroscopy in dielectric microchips. The Analyst, 139(12):3115-21, 2014. ISSN 1364-5528. doi: 10.1039/c4an00212a. URL http://www.ncbi.nlm.nih.gov/pubmed/24781384. 
[24] A.V. Mamishev, K. Sundara-Rajan, and M. Zahn. Interdigital sensors and transducers. Proceedings of the IEEE, 92(5): 808-845, May 2004. ISSN 0018-9219. doi: 10.1109/JPROC.2004.826603. URL http://ieeexplore.iee.org/lpdocs/epic03/ wrapper.htm?arnumber $=1288505$.

[25] Joseph Wang. Analytical electrochemistry. John Wiley \& Sons, Inc., Hoboken, New Jersey, 3 edition, 2006.

[26] Zhiwei Zou, Junhai Kai, Michael J. Rust, Jungyoup Han, and Chong H. Ahn. Functionalized nano interdigitated electrodes arrays on polymer with integrated microfluidics for direct bio-affinity sensing using impedimetric measurement. Sensors and Actuators A: Physical, 136(2):518-526, May 2007. ISSN 09244247. doi: 10.1016/j.sna.2006.12.006. URL http://linkinghub.elsevier.com/retrieve/pii/S0924424706007588.

[27] Gerald M. Birnbaumer, Peter A. Lieberzeit, Lukas Richter, Romana Schirhagl, Marcus Milnera, Franz L. Dickert, Andrew Bailey, and Peter Ertl. Detection of viruses with molecularly imprinted polymers integrated on a microfluidic biochip using contact-less dielectric microsensors. Lab on a chip, 9(24):3549-56, December 2009. ISSN 1473-0197. doi: 10.1039/b914738a. URL http://www.ncbi.nlm.nih.gov/pubmed/20024035.

[28] Kosuke Ino, Yusuke Kitagawa, Tsuyoshi Watanabe, Hitoshi Shiku, Masahiro Koide, Tomoaki Itayama, Tomoyuki Yasukawa, and Tomokazu Matsue. Detection of hormone active chemicals using genetically engineered yeast cells and microfluidic devices with interdigitated array electrodes. Electrophoresis, 30(19):3406-12, October 2009. ISSN $1522-2683$. doi: 10.1002/elps.200900244. URL http://www.ncbi.nlm.nih.gov/pubmed/19802852.

[29] Gerardus Rudolph Langereis. An integrated sensor system for monitoring washing processes. PhD thesis, Universiteit Twente, 1999.

[30] Björn Timmer, Wouter Sparreboom, Wouter Olthuis, Piet Bergveld, and Albert van den Berg. Optimization of an electrolyte conductivity detector for measuring low ion concentrations. Lab on a chip, 2(2):121-4, May 2002. ISSN 1473-0197. doi: 10.1039/b201225a. URL http://www.ncbi.nlm.nih.gov/pubmed/15100845.

[31] Jongin Hong, Dae Sung Yoon, Sung Kwan Kim, Tae Song Kim, Sanghyo Kim, Eugene Y. Pak, and Kwangsoo No. AC frequency characteristics of coplanar impedance sensors as design parameters. Lab on a chip, 5(3):270-9, March 2005. ISSN 1473-0197. doi: 10.1039/b410325d. URL http://www.ncbi.nlm.nih.gov/pubmed/15726203.

[32] Mouhamad Ibrahim, Julien Claudel, Djilali Kourtiche, and Mustapha Nadi. Geometric parameters optimization of planar interdigitated electrodes for bioimpedance spectroscopy. Journal of Electrical Bioimpedance, 4(1):13-22, March 2013. ISSN 1891-5469. doi: 10.5617/jeb.304. URL https://www.journals.uio.no/index.php/bioimpedance/article/view/304.

[33] Muinul H. Banna, Homayoun Najjaran, Rehan Sadiq, Syed A. Imran, Manuel J. Rodriguez, and Mina Hoorfar. Miniaturized water quality monitoring pH and conductivity sensors. Sensors and Actuators, B: Chemical, 193:434-441, 2014. ISSN 09254005. doi: 10.1016/j.snb.2013.12.002. URL http://dx.doi.org/10.1016/j.snb.2013.12.002.

[34] Wendell Karlos Tomazelli Coltro, Rodrigo De Santis Neves, Artur De Jesus Motheo, José Alberto Fracassi Da Silva, and Emanuel Carrilho. Microfluidic devices with integrated dual-capacitively coupled contactless conductivity detection to monitor binding events in real time. Sensors and Actuators, B: Chemical, 192:239-246, 2014. ISSN 09254005. doi: 10.1016/j.snb.2013.10.114. URL http://dx.doi.org/10.1016/j.snb.2013.10.114. 
[35] Christian Jungreuthmayer, Gerald M Birnbaumer, Juergen Zanghellini, and Peter Ertl. 3D numerical simulation of a labon-a-chip-increasing measurement sensitivity of interdigitated capacitors by passivation optimization. Lab on a chip, 11 (7):1318-25, April 2011. ISSN 1473-0189. doi: 10.1039/c0lc00543f. URL http://www.ncbi.nlm.nih.gov/pubmed/21331426.

[36] Rui Igreja and C.J. Dias. Analytical evaluation of the interdigital electrodes capacitance for a multi-layered structure. Sensors and Actuators A: Physical, 112(2-3):291-301, May 2004. ISSN 09244247. doi: 10.1016/j.sna.2004.01.040. URL http://linkinghub.elsevier.com/retrieve/pii/S0924424704000779.

[37] Rui Igreja and C.J. Dias. Extension to the analytical model of the interdigital electrodes capacitance for a multi-layered structure. Sensors and Actuators A: Physical, 172(2):392-399, December 2011. ISSN 09244247. doi: 10.1016/j.sna.2011. 09.033. URL http://linkinghub.elsevier.com/retrieve/pii/S0924424711005474.

[38] Steffen O.P. Blume, Ridha Ben-Mrad, and Pierre E. Sullivan. Modelling the capacitance of multi-layer conductor-facing interdigitated electrode structures. Sensors and Actuators B: Chemical, 213:423-433, 2015. ISSN 09254005. doi: 10.1016/j.snb.2015.02.088. URL http://linkinghub.elsevier.com/retrieve/pii/S0925400515002737.

[39] Wei Lai. Impedance Spectroscopy as a Tool for the Electrochemical Study of Mixed Conducting Ceria. PhD thesis, California Institute of Technology, 2007.

[40] Yuriy Shmaliy. Continuous-time systems. Springer, Dordrecht, The Netherlands, 2007.

[41] MatWeb.com. Corning Pyrex 7740 Borosilicate Glass, 2014. URL http://matweb.com/search/datasheet.aspx?MatGUID= 5bb651ca58524e79a503011b2cd8083d.

[42] Specialty Coating Systems. SCS Parylene Properties, 2007. URL http://scscoatings.com/corporate/library.aspx.

[43] Amorphous Fluoropolymer Comparison, DuPont Teflon AF, 2014. URL http://www2.dupont.com/Teflon_Industrial/ en_US/products/product_by_name/teflon_af/performance.html.

[44] Jospeh C. Salamone. Polymeric Materials Encyclopedia. CRC Press, Inc., Boca Raton, Florida, USA, 1996.

[45] Stephen Bone, Joyce Eden, and Ronald Pethig. Electrical Properties of Proteins as a Function of Hydration and NaCl Content. International Journal of Quantum Chemistry: Quantum Biology Symposium, 8:307-316, 1981.

[46] Zbigniew Stojek. The Electrical Double Layer and Its Structure. In Fritz Scholz, editor, Electroanalytical Methods: Guide to Experiments and Applications, chapter I.1, pages 3-8. Springer-Verlag, Berlin, Heidelberg, Germany, 2 edition, 2010.

[47] Hanbin Ma, Richard W R Wallbank, Reza Chaji, Jiahao Li, Yuji Suzuki, Chris Jiggins, and Arokia Nathan. An impedance-based integrated biosensor for suspended DNA characterization. Scientific reports, 3:2730, January 2013. ISSN 2045-2322. doi: 10.1038/srep02730. URL http://www.pubmedcentral.nih.gov/articlerender.fcgi?artid=3781400\& tool $=$ pmcentrez\&rendertype $=$ abstract.

[48] Allen J. Bard and Larry R. Faulkner. Electrochemical Methods: Fundamentals and Applications. John Wiley \& Sons, Inc., New York, 2001. ISBN 0471043729. 
[49] Christopher M.A. Brett and Ana Maria Olivera Brett. Electrochemistry: Principles, Methods, and Applications. Oxford University Press, Oxford, New York, Tokyo, 1993.

[50] Vladimir S. Bagotsky. Fundamentals of electrochemistry. John Wiley \& Sons, Inc., Hoboken, New Jersey, 2nd edition, 2006. ISBN 978-0-471-70058-6.

[51] Nicholas G. Green. Electrostatics and Quasielectrostatics. In Antonio Ramos, editor, Electrokinetics and Electrohydrodynamics in Microsystems, pages 29-59. Springer, Wien, New York, 2011. ISBN 978-3-7091-0899-4. URL http://link.springer.com/chapter/10.1007/978-3-7091-0900-7_2.

[52] Petr Vanysek. CRC Handbook of Chemistry and Physics. CRC Press, Inc., Boca Raton, Florida, USA, 95 edition, 2014. URL http://www.hbcpnetbase.com/.

[53] Christine Lefrou, Pierre Fabry, and JC Poignet. Electrochemistry: The Basics, with Examples. Springer, Berlin Heidelberg, 2012. ISBN 9782759804252. URL http://books.google.com/books?hl=en\&lr=\&id=Z_5rBvypSwAC\&oi=fnd\&pg=PR5\& $\mathrm{dq}=$ Electrochemistry, + The+basics, + with + examples\&ots=Zp0JC0JSRv\&sig=q1yhN9GdMmLUKdKpWTlwjatQcfI.

[54] Sigma Aldrich. Potassium chloride solution, 1.0 M conductance standard, 2014. URL http://www.sigmaaldrich.com/ catalog/product/fluka/60131?lang=en\&region=CA. 


\section{Appendices}




\section{A The Debye length}

The Debye length $\kappa^{-1}$ is given by $[48,49]$

$$
\kappa^{-1}=\sqrt{\frac{\varepsilon_{r} \varepsilon_{0} k_{B} T}{2 \times 10^{3} n_{j} z_{j}^{2} e^{2}}}
$$

In Equation (4), $\varepsilon_{r}$ is the relative permittivity of the liquid, $\varepsilon_{0}$ is the permittivity of vacuum, $k_{B}$ is the Boltzmann constant, $T$ is the absolute temperature, $z_{j}$ is the magnitude of charge on the ions, and $e$ is the elementary charge. The number concentration $n_{j}$ is related to the molar concentration by $n_{j}=c_{j} N_{A}$, where $N_{A}$ is Avogadro's constant. Since $\kappa^{-1}$ is typically given in $(\mathrm{cm})$, Equation (4) includes a factor of $10^{3}$. For a symmetric binary electrolyte solution $(z=1)$, such as potassium chloride (KCl) in water, and an electrolyte concentration of $0.0001 \mathrm{M}$, the Debye length is $30.6 \mathrm{~nm}$ at $T=295 \mathrm{~K}$. 


\section{B Conductivities of $\mathrm{KCl}$ solutions}

For a binary and symmetric (1:1) electrolyte solution the solution conductivity can be determined according to $[50,51]$

$$
\sigma=\Lambda c_{k}
$$

where $\Lambda$ denotes the molar conductivity for a symmetric (binary) electrolyte solution. For low electrolyte concentrations $\left(\leq 10^{-3} \mathrm{~mol} \cdot \mathrm{L}^{-1}=1.0 \mathrm{mM}\right)$, the molar conductivity is approximated by the Debye-HückelOnsager equation [52],

$$
\Lambda=\Lambda^{0}-K_{\Lambda} \sqrt{c_{k}}=\Lambda^{0}-\left(A+B \Lambda^{0}\right) \sqrt{c_{k}}
$$

where the constant $K_{\Lambda}$ as well as $\Lambda^{0}$ are dependent on the thermodynamic state of the electrolyte solution [53]. The parameter $\Lambda^{0}$ is the ideal molar conductivity at infinite dilution. For a binary, symmetric electrolyte solution at $25^{\circ} \mathrm{C}$, the constants are $A=60.20$ and $B=0.229$. The following table gives the molar conductivity and solution conductivity for a solution of various concentrations of $\mathrm{KCl}$ in water.

Table 3 Conductivity of $\mathrm{KCl}$ solutions at different molarities at $25^{\circ} \mathrm{C}$. Most values are gathered from [52], unless otherwise noted. According to Equation (6) the conductivity for a $0.0001 \mathrm{M} \mathrm{KCl}$ solution is

\begin{tabular}{|c|c|c|c|c|}
\hline & \multicolumn{4}{|c|}{ Concentration $(\mathrm{M}=\mathrm{mol} / \mathrm{L})$} \\
\hline Infinite dilution & 0.001 & 0.01 & 0.1 & 1.0 \\
\hline$\Lambda^{0}$ & \multicolumn{4}{|c|}{$\Lambda\left(10^{-4} \mathrm{~m}^{2} \mathrm{~S} \mathrm{~mol}^{-1}\right)$} \\
\hline \multirow[t]{5}{*}{149.49} & 146.88 & 141.20 & 128.90 & - \\
\hline & $\Lambda\left(10^{-4} \mathrm{r}\right.$ & ${ }^{2} \mathrm{~S} \mathrm{~mol}^{-1}$ & using $\mathrm{I}$ & uation $(6)$ \\
\hline & 146.50 & 140.05 & 119.63 & 55.06 \\
\hline & \multicolumn{4}{|c|}{$\sigma(\mathrm{S} / \mathrm{m})$} \\
\hline & 0.014688 & 0.1412 & 1.289 & $11.18[54]$ \\
\hline
\end{tabular}
$1.4855 \times 10^{-3} \mathrm{~S} / \mathrm{m}$. 